\title{
Maximization of Received Signal Power by Impedance Matching in Human Body Communication Receiver
}

\author{
Naruto Arai Dairoku Muramatsu \\ Department of Human and Engineered Environmental Studies \\ Graduate School of Frontier Science, The University of Tokyo \\ 5-1-5, Kashiwanoha, Kashiwa-shi, Chiba 277-8563, JAPAN \\ +81-4-7136-4618, JP \\ narai@ems.k.u-tokyo.ac.jp \\ muramatsu@ems.k.u- \\ tokyo.ac.jp \\ Ken Sasaki \\ ksasaki@k.u-tokyo.ac.jp
}

\begin{abstract}
Human body communication (HBC) utilizes human body as part of the transmission channel. The present paper deals with HBC between a transmitter worn on the user's wrist and an off-body stationary receiver touched by the user's finger. In this configuration, transmitter-human-body system can be regarded as a signal source as viewed from the receiver. The equivalent output impedance of this signal source mainly consists of resistance of human body and capacitive reactance among the receiver, human body, and the surrounding environment. Impedance matching was achieved by inserting an inductor to cancel the capacitive reactance and adjusting the input resistor to match the resistance of the equivalent output impedance. At frequency of $10 \mathrm{MHz}$, maximum received power was obtained with $40 \mu \mathrm{H}$ inductor and $1.5 \mathrm{k} \Omega$ input resistance. This suggests that the output impedance of the transmitter-human-body system is $1500-\mathrm{j} 2500 \Omega$.
\end{abstract}

\section{Categories and Subject Descriptors}

H.5.2 [User Interfaces]: Input devices and strategies

\section{General Terms}

Measurement, Experimentation

\section{Keywords}

Human Body Communication, Human Interface, Impedance Matching

\section{INTRODUCTION}

Human body communication (HBC) is a wireless transmission method for wearable devices which uses human body as a part of the signal transmission channel. One of the early studies on HBC is the report by Zimmerman in 1995 [1]. In 2012, IEEE 802.15.6 standard was accepted for Body Area Network, and HBC was adopted as one of the physical layers of short-range radio communication networks around human body [2]. This standard states that $\mathrm{HBC}$ uses electric field communication (EFC) technology and its operation is centered at $21 \mathrm{MHz}$. This standard, however, does not provide technical details on EFC technology. EFC technology is relatively new and it is not studied as much as other wireless communication technologies. Engineers who intend to use EFC technology have limited information on circuit design. The present study aims to provide some technical information for designing $\mathrm{HBC}$ devices.

HBC uses electrodes that are in contact with or placed close to the human body. These electrodes are the equivalent of antennas in wireless communication. The difference is that airborne antennas transmit electromagnetic wave into the air, while electrodes in $\mathrm{HBC}$ generate electric field around and inside the human body. One of the design parameters that we need to know for circuit design is the impedance characteristics of electrodes used in HBC. Although previous studies on electrode impedance characteristics have been studied by electromagnetic analysis and by experiments using phantoms $[3,4,5]$, we need quantitative data on impedance characteristics measured by using real human body.

There are three possible forms of HBC: 1) communication among multiple devices worn by a single person, 2) communication between devices worn by two people that have physical contact such as shaking hands, and 3) communication between a wearable device and an off-body stationary device that is touched by the user. This study focuses on the third form, more specifically, data transmission from a wearable device worn on the user's wrist to an off-body stationary device touched by the user's index finger. Application examples of such data transmission are, going through a ticket gate, or payment with electronic money at vending machines.

Transmission between a transmitter worn on the user's wrist and an off-body stationary receiver touched by the user's index finger consists of current flow through the finger and arm, and current flow through capacitive coupling between human body and receiver's outer case, which is usually made of metal, and connected to the ground of the receiver circuit. Figure 1 shows the simplified equivalent circuit of this transmission. $Z_{r}$ is the input impedance of receiver circuit, and $Z_{h}$ is the equivalent output impedance of the signal source which is the transmitter-humanbody system. The signal electrode of the receiver has physical contact with the human body, while the ground electrode, which is the outer case of the receiver, has no contact with the human body. It is electrically connected by capacitive coupling with the human body. It is known that capacitive coupling among the receiver, the human body and the surroundings affect the communication characteristic $[6,7,8]$. Therefore, we hypothesize that the equivalent output impedance $Z_{h}$ is composed of capacitive reactance and some resistance of the human body. 
One of the common circuit design guidelines is to maximize received signal power by matching the input impedance to the output impedance of the signal source. In the following experiments, we first insert an inductor between the receiver electrode and the input terminal of the receiver to cancel the capacitive reactance and verify that capacitive reactance exists in the transmission path. Next, the input resistance of the receiver is varied to find the resistance that gives maximum received signal power. The matched inductance and resistance corresponds to the equivalent capacitive reactance and resistance of the transmitterhuman-body signal source.

\section{TRANSMITTER AND RECEIVER}

Figure 2 shows the experimental setup. A subject wore a transmitter on his wrist and touched the receiver electrode with his index finger. The transmitter is a battery operated Colpitts oscillator that outputs $10 \mathrm{MHz} 1.0 \mathrm{Vpp}$ sine wave across the two transmitter electrodes that are in contact with the skin of the wrist. Frequency of $10 \mathrm{MHz}$ was selected according to the previous research that reported that frequency around $10 \mathrm{MHz}$ was suited for HBC [9]. Figure 3 shows the placement of electrodes and circuit board with respect to the arm. Three types of electrode configurations were prepared as shown in Fig. 4. All electrodes were $24 \times 8 \mathrm{~mm}$ in size. The distances between the two electrodes were $8 \mathrm{~mm}, 24 \mathrm{~mm}$, and $40 \mathrm{~mm}$ respectively. Circuit diagram of the receiver is shown in Fig. 5. Receiver electrode was made of stainless steel sheet and its size was $5 \times 5 \mathrm{~mm}$. Received signal was amplified by two-stage non-inverting amplifiers and a band pass. Total gain was $29.2 \mathrm{~dB}$. Output amplitude was measured by a battery operated oscilloscope. This receiver circuit was placed in an aluminum box ( $200 \times 150 \times 40 \mathrm{~mm})$, and the circuit ground was connected to this aluminum box near the receiver electrode. The receiver and the oscilloscope were placed on a wooden testing stand. Photograph of the experimental setup is shown in Fig. 6. Input impedance of the receiver circuit was first set at 4.7 $\mathrm{k} \Omega$, and was changed to trimmer resistor in the second experiment. In this experimental setup, shield of the coaxial cable and the oscilloscope also contribute to the capacitive coupling. It should be noted that large part of the capacitive coupling is formed around the arm and the receiver and the oscilloscope. Capacitive coupling with other parts of the body and ground loop through the floor have little contribution to the signal transmission [5]. Experiments involving measurement using human body in this study have been approved by Research Ethics Committee of the University of Tokyo.

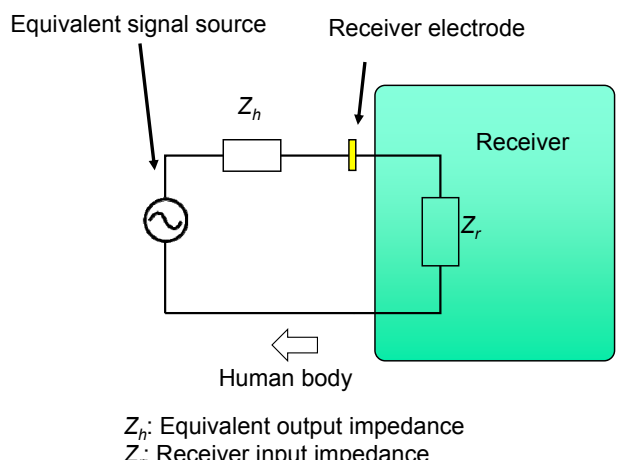

Figure 1. Equivalent circuit of HBC transmission.
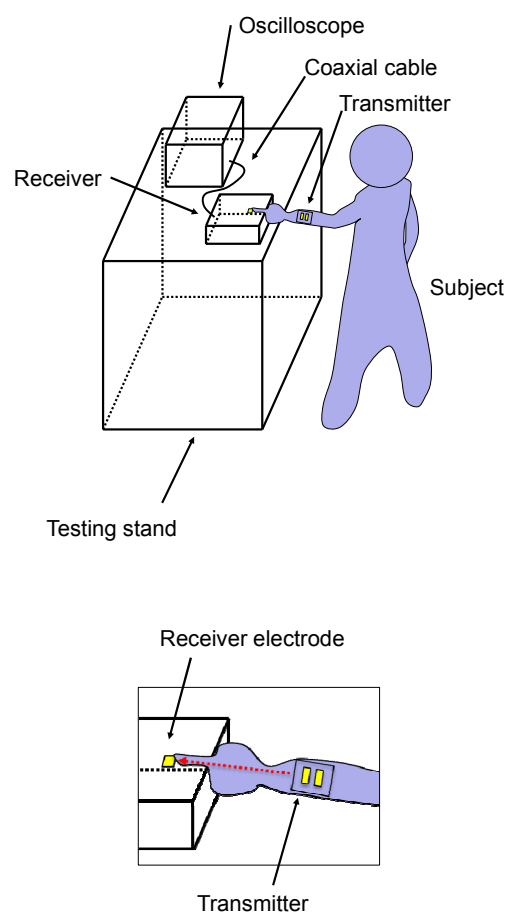

Figure 2. Experimental setup.

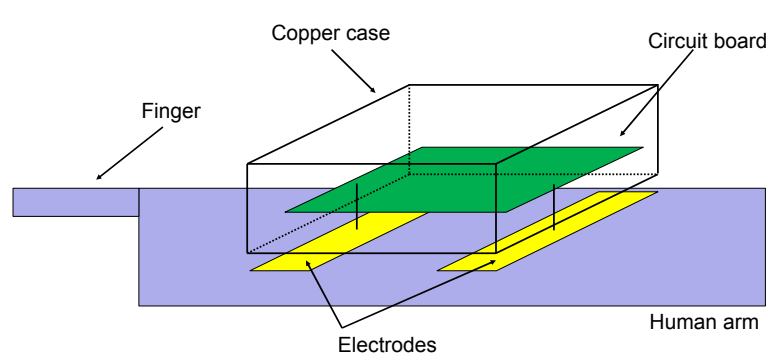

Figure 3. Placement of electrodes and circuit board.
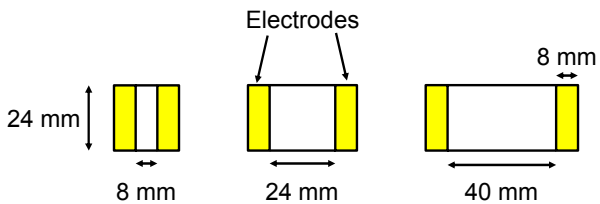

Figure 4. Configurations of transmitter electrodes. 


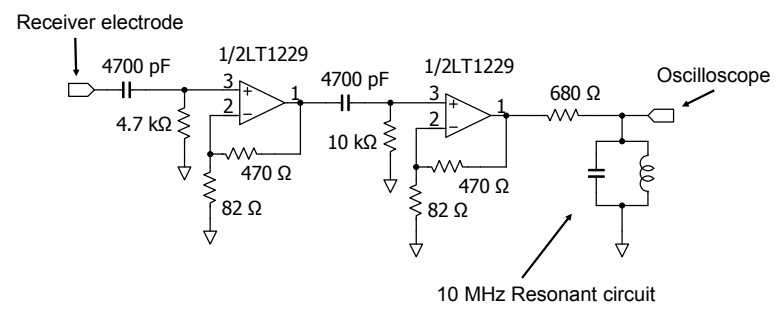

Figure 5. Circuit diagram of receiver circuit.

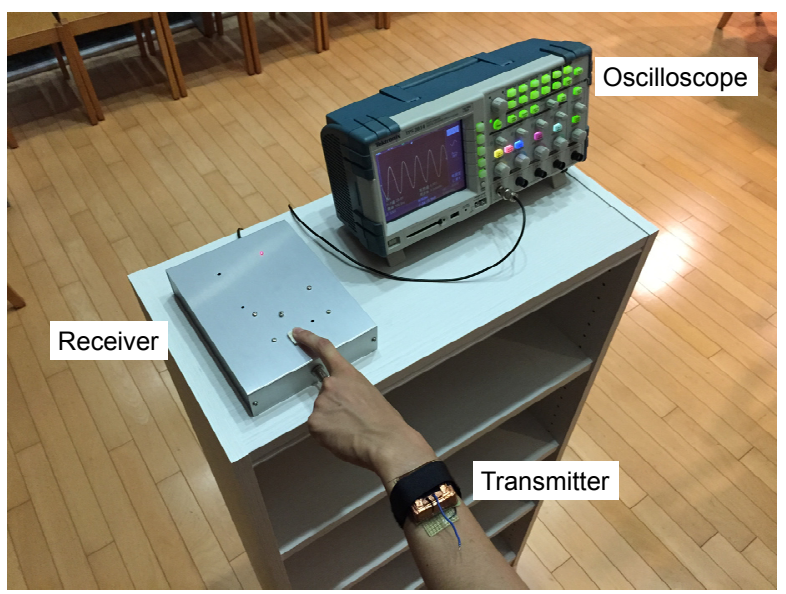

Figure 6. Experimental setup (photograph).

\section{IMPEDANCE MATCHING OF REACTANCE}

An inductor was inserted between the receiver electrode and the receiver circuit, and the output voltage of the receiver output was measured. The imaginary part of the equivalent output impedance was expected to be in the order of several thousands of Ohm based on our previous studies (not published). Several inductors ranging from $1 \mu \mathrm{H}$ to $86 \mu \mathrm{H}$, which correspond to impedances of $63 \Omega$ to $5400 \Omega$, were inserted between the receiver electrode and the input of the receiver circuit. Instead of using nominal values shown on the parts, effective inductances measured by an impedance analyzer at $10 \mathrm{MHz}$ were used for calculation. For example, an inductor whose nominal value was $22 \mu \mathrm{H}$ had an inductance of $40 \mu \mathrm{H}$ at $10 \mathrm{MHz}$. The received power was calculated from the received voltage and the input impedance (4.7 $\mathrm{k} \Omega$ ) of the receiver.

Figure 7 shows the result of this measurement. The largest received signal powers were $272 \mathrm{nW}, 419 \mathrm{nW}$, and $485 \mathrm{nW}$, when distances between the two transmitter electrodes were $8 \mathrm{~mm}, 24$ $\mathrm{mm}$, and $40 \mathrm{~mm}$ respectively. Received signal maxima were obtained when an inductor of $40 \mu \mathrm{H}$ was inserted for all three electrode configurations. This result suggests that capacitive reactance exists in the transmission path, and that this capacitive reactance was canceled by the inductor. Furthermore, this capacitive reactance was not affected by the transmitter electrode configuration. Impedance of $40 \mu \mathrm{H}$ at $10 \mathrm{MHz}$ is $2500 \Omega$. This corresponds to a capacitance of approximately $6.3 \mathrm{pF}$.

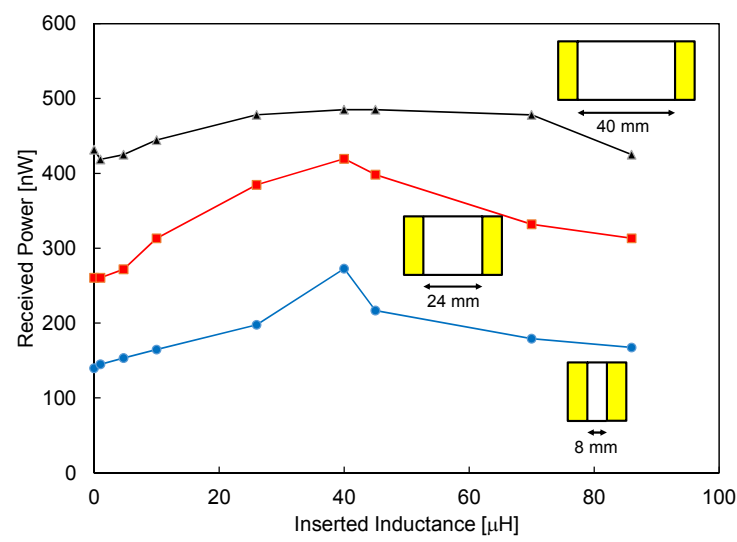

Figure 7. Relationship between inserted inductance and received power.

\section{IMPEDANCE MATCHING OF RESISTANCE}

In the previous experiment, the input impedance of the receiver was fixed at $4.7 \mathrm{k} \Omega$. Next, this resistor was replaced by a trimmer resistor to change the input impedance of the receiver to find a resistance that gave maximum signal power. This resistance will be the resistive component of the equivalent output impedance of the signal source. An inductor of $40 \mu \mathrm{H}$ was inserted to cancel the capacitive reactance according to the result of the previous experiment. Figure 8 shows the equivalent circuit of this impedance matching. Received voltage was measured by setting the resistance of the trimmer resistor to eleven different values ranging from $200 \Omega$ to $4.7 \mathrm{k} \Omega$. Figure 9 shows the result. The largest received powers were $337 \mathrm{nW}, 635 \mathrm{nW}$, and $994 \mathrm{nW}$ when the distances between the two transmitter electrodes were $8 \mathrm{~mm}$, $24 \mathrm{~mm}$, and $40 \mathrm{~mm}$ respectively. Received power was at its maximum when the resistance was approximately $1.5 \mathrm{k} \Omega$ for all three electrode configurations. This means that the real part of the equivalent output impedance is not changed by the transmitter electrode configuration. By combining the results of the two experiments, we estimated that the output impedance of the transmitter-human-body system was $1500-\mathrm{j} 2500 \Omega$ at $10 \mathrm{MHz}$. This is equivalent to $6.3 \mathrm{pF}$ capacitance and $1.5 \mathrm{k} \Omega$ resistance in series.

This result indicates that received signal power can be maximized by setting the input impedance of the receiver to matched impedance of $1500+\mathrm{j} 2500 \Omega$. However, this does not mean that the actual front end of the receiver circuit should be the circuit shown in Fig. 8.

Table 1 shows comparison of received power under three conditions: 1)without impedance matching ( $4.7 \mathrm{k} \Omega$ input resistor only), 2)cancelling capacitive reactance only (inserting $40 \mu \mathrm{H}$ inductor), and 3)matching both the imaginary and the real part (using $40 \mu \mathrm{H}$ inductor and $1.5 \mathrm{k} \Omega$ resistor). The received power obtained with matched impedance was approximately twice as large as that obtained without impedance matching. 
Table 1. Relationship between received power and impedance matching

\begin{tabular}{|l|l|l|l|}
\hline & \multicolumn{3}{|l|}{ Distance between two transmitter electrodes } \\
\hline & $8 \mathrm{~mm}$ & $24 \mathrm{~mm}$ & $40 \mathrm{~mm}$ \\
\hline Without impedance matching & $140 \mathrm{nW}$ & $261 \mathrm{nW}$ & $432 \mathrm{nW}$ \\
\hline Cancelling capacitive reactance only & $272 \mathrm{nW}$ & $419 \mathrm{nW}$ & $485 \mathrm{nW}$ \\
\hline Matching both the imaginary and real part & $337 \mathrm{nW}$ & $635 \mathrm{nW}$ & $994 \mathrm{nW}$ \\
\hline
\end{tabular}

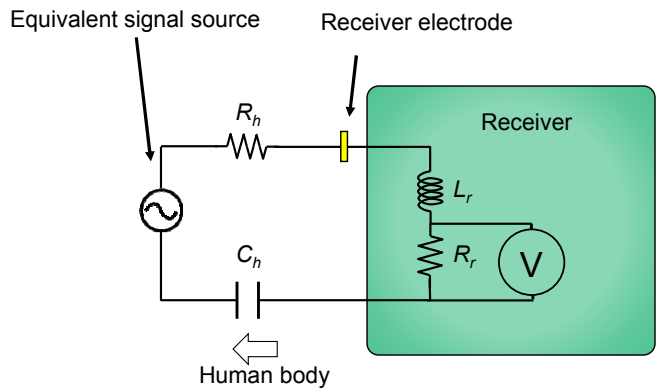

$R_{h}, C_{h}$ : Equivalent output impedance $R_{r}, L_{r}$ : Receiver input impedance

Figure 8. Equivalent circuit of impedance matching.

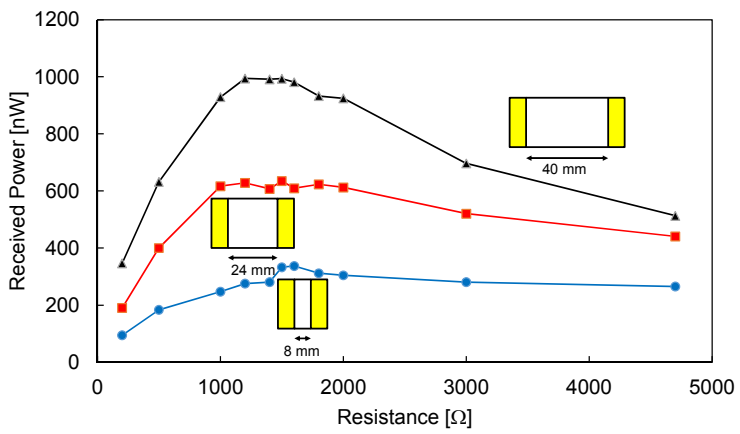

Figure 9. Relationship between input resistance and received power.

\section{CONCLUSION}

Impedance matching of $\mathrm{HBC}$ between a wearable transmitter and an off-body receiver touched by user's index finger was investigated. Transmitter-human-body system was modelled as a signal source in the equivalent circuit model of this transmission. Impedance matching of equivalent output impedance was achieved by inserting an inductor to cancel the capacitive reactance and adjusting the input resistor to match the real part of the equivalent output impedance. The equivalent output impedance was $1500-\mathrm{j} 2500 \Omega$ at $10 \mathrm{MHz}$. This value can be used to design an efficient front end circuit of the HBC receiver.

\section{ACKNOWLEDGMENTS}

Part of this work was supported by Japan Society for Promotion of Science (JSPS) Grants-in-Aid for Scientific Research 26420340 .

\section{REFERENCES}

[1] Zimmerman, T.G. 1995. Personal area networks (PAN): near-field intra-body communication. M.S. thesis, MIT media laboratory, 1995

[2] B IEEE standard for local and metropolitan area networkspart 15.6: wireless body area networks. IEEE Computer Society, 2012

[3] Fujii, K., Takahashi, M. and ITO, K. 2007. Electric field distributions of wearable devices using the human body as a transmission channel. IEEE transactions on antennas and propagation, 55 (7). 2080-2087.

[4] Fujii, K., Takahashi, M., Ito, K., Hachisuka, K., Terauchi, Y., Kishi, Y., Sasaki, K. and Itao, K. 2005. Study on the transmission mechanism for wearable device using the human body as a transmission channel. IEICE transactions on communications, E88-B (6). 2401-2410.

[5] Muramatsu, D., Koshiji, F., Koshiji, K. and Sasaki, K. 2014. Analytical and experimental studies on human body communication between wristwatch and handheld device using muscle homogenous phantom at $10 \mathrm{MHz}$. Sensors and materials, 26 (8). 581-589.

[6] Baldus, H., Corroy, S., Fazzi, A., Klabunde, K. and Schenk, T. 2009. Human-centric connectivity enabled by bodycoupled communications. IEEE communications magazine, 47 (6). 172-178

[7] Partridge, K., Dahlquist, B., Veiseh, A., Cain, A., Foreman, A., Goldberg, J. and Borriello, G. 2001. Empirical measurements of intrabody communication performance under varied physical configurations. in The 14th Annual ACM Symposium on User Interface Software and Technology, (Florida, U.S., 2001), Association for Computing Machinery, Inc., 183-190.

[8] Xu, R., Ng, W.C., Zhu, H., Shan, H. and Yuan, J. 2012. Equation environment coupling and interference on the electric-field intrabody communication channel. IEEE Transactions on biomedical engineering, 59 (7). 2051-2059.

[9] Hachisuka, K., Takeda, T., Terauchi, Y., Sasaki, K., Hosaka., $\mathrm{H}$. and Itao, K. Intra-body data transmission for the personal area network. Microsystem technologies, 11 (8-10), 10201027. 